\title{
PERFIL METABÓLICO, HEMATOLÓGICO E COMPORTAMENTAL DE POEDEIRAS SUPLEMENTADAS COM PANAX GINSENG
}

\author{
METABOLIC, HEMATOLOGICAL AND BEHAVIORAL PROFILE OF LAYING HENS \\ SUPPLEMENTED WITH PANAX GINSENG
}

\author{
Catalan, A.A.S. ${ }^{1 *}$; Avila, V.S. ${ }^{2}$; Lopes, L.L. ${ }^{1}$; Montagner, P. ${ }^{1}$; Vargas, G.D. ${ }^{3}$; Xavier, E.G. ${ }^{1}$ \\ e Roll, V.F.B. ${ }^{1}$
}

1DZ/FAEM. Universidade Federal de Pelotas. Pelotas, RS. Brasil. *aianec@yahoo.com.br EMBRAPA Suínos e Aves. Concórdia, SC. Brasil.

${ }^{3}$ Departamento de Veterinária Preventiva. Universidade Federal de Pelotas (UFPel). Pelotas, RS. Brasil.

\section{PalaVRas ChaVe adicionaIS \\ Aditivo fitogênico. Bem-estar. Fisiologia.}

\section{RESUMO}

Neste trabalho foram avaliados o perfil hematológico, bioquímico e comportamental de poedeiras recebendo um produto comercial a base de Panax ginseng na fase inicial de postura. As dietas experimentais foram fornecidas as aves durante 30 dias. O delineamento experimental foi inteiramente casualizado com cinco tratamentos que consistiram em diferentes doses de $P$. ginseng (T1: controle, sem $P$. ginseng; 2 : 1,9 mg/ave/dia; T3:3,8 mg/ave/dia; T4:5,7 mg/ave/ dia; T5: 7,6 mg/ave/dia) num total de 70 aves, distribuídas em 35 gaiolas, divididas em sete repetições por tratamento. As dietas basais foram formuladas a base de milho e farelo de soja, atendendo as exigências das aves. Para predizer os efeitos das doses de $P$. ginseng sobre os parâmetros metabólicos e hematológicos foi utilizada análise de regressão polinomial. Foi utilizado o teste de Dunnett, em cada nível de inclusão do $P$. ginseng, para comparação com a ração controle. Para a análise da frequência de comportamentos foi utilizado o teste de qui-quadrado. No perfil hematológico das poedeiras não foram observadas diferenças significativas entre os tratamentos em nenhuma das fases analisadas. No perfil metabólico foram observados maiores níveis de fósforo nas aves que receberam 7,6 mg/ave/dia de $P$. ginseng em comparação com o grupo controle nos períodos dois e três de avaliação e de albumina somente neste último período. Os níveis de colesterol foram maiores nas aves recebendo

\author{
AdDITIONAL KEYWORDS \\ Physiology. Phytogenic additive. Welfare.
}

dosagens de 1,9 e $3,8 \mathrm{mg} /$ ave/dia de $P$. ginseng em relação ao grupo controle. Nas variáveis etológicas o comportamento de vigiar foi mais frequente no grupo controle, o comportamento de toilette ou higiene no grupo com a maior dose de $P$. ginseng $(7,6 \mathrm{mg} / \mathrm{ave} / \mathrm{dia})$ e beber água nas doses intermediárias (3,8 e 5,6 mg/ave/dia). A suplementação com $P$. ginseng até $7,6 \mathrm{mg} / \mathrm{ave} / \mathrm{dia}$ não alterou o perfil hematológico. Apenas pequenas variações no perfil metabólico e na frequência de alguns comportamentos podem ser esperadas com o uso do $P$. ginseng.

\section{SUMMARY}

The aim of this study was to evaluate the biochemical, hematological and behavioral profile of hens supplemented with a commercial Panax ginseng product during initial hen's laying cycle. The experimental design was completely randomized with five treatments which consisted in different levels of $P$. ginseng (T1: control, without $P$. ginseng; T2: $1.9 ;$ T3: $3.8 ;$ T4: 5.7 and T5: $7.6 \mathrm{mg} / \mathrm{hen} /$ day) in a total of 70 birds housed in 35 cages with seven replicates per treatment. The basal diets were prepared using corn and soybean meal in agreement with the nutritional values established by the breeder manual. To predict the effect of different levels of $P$. ginseng on biochemical and hematological traits the regression analysis was used. The Dunnett test was used in 


\section{CATALAN, AVILA, LOPES, MONTAGNER, VARGAS, XAVIERE ROLL}

each $P$. ginseng levels to compare to the control treatment. Ethological parameters were evaluated using chi-square analysis. No significant difference was found in hen's hematological profile in all periods evaluated. In metabolic parameters was observed higher level of phosphorus in hens receiving $7.6 \mathrm{mg} / \mathrm{hen} /$ day of $P$. ginseng at second and third period and albumin was observed only in the last period when compared to the control group. Cholesterol level was higher in hens receiving 1.9 and $3.8 \mathrm{mg} / \mathrm{hen} /$ day of $P$. ginseng in comparison with the control treatment. In ethological parameters vigilant behavior was more frequent in control group, preening in the group receiving $7.6 \mathrm{mg} / \mathrm{hen} /$ day and drinking in the intermediate levels of $P$. ginseng ( 3.8 and $5.6 \mathrm{mg} / \mathrm{hen} /$ day). The supplementation with $P$. ginseng up to $7.6 \mathrm{mg} / \mathrm{hen} /$ day do not change the hematological profile. Only small variations in the metabolic parameters and behavior frequency of the hens receiving ginseng could be expected.

\section{INTRODUÇÃO}

O Panax ginseng é uma planta medicinal, qual é reconhecida mundialmente como a planta que cura todos os males (Choi, 2008). A partir dos princípios ativos presentes na raiz desta planta são elaborados os medicamentos fitoterápicos, que segundo Kiefer e Pantuso (2003) possuem uma variedade de efeitos benéficos, incluindo anti-inflamatório, antioxidante, anticancerígenos. Porém, na avicultura suas propriedades ainda são pouco conhecidas. Por exemplo, Lee et al. (2008) observaram que a suplementação com 0,5 e $1 \%$ de ginseng siberiano aumentava, a altura de albúmen, a unidade Haugh e o peso dos ovos. Park et al. (2005) também observaram efeitos positivos de subprodutos de ginseng através do aumento da produção e massa de ovos e na espessura da casca nos níveis 0,$4 ; 0,8$; 1,6 e 3,2 \% de suplementação na dieta. De forma semelhante, Jang et al. (2007) encontraram maior peso dos ovos, altura de albúmen, unidade Haugh e coloração da gema quando as dietas foram suplementadas com $5,0 \%$, de um subproduto fermentado de ginseng.
Desta forma, objetivou-se neste trabalho caracterizar a ação do $P$. ginseng sobre o perfil hematológico, metabólico e comportamental de poedeiras na fase inicial da postura.

\section{MATERIALEMÉTODOS}

Foram utilizadas 14 aves por tratamento, totalizando 70 poedeiras da linhagem Hisex Brown, com 21 semanas de idade durante 60 dias experimentais. Estas foram alojadas em gaiolas piramidais, com comedouro individual do tipo calha, colocadas externa e longitudinalmente na frente da gaiola, além de bebedouro do tipo nipple localizado no centro de cada gaiola.

As aves foram alimentadas com dietas à base de milho, farelo de soja, farelo de arroz desengordurado, farinha de ostra, sal iodado, suplemento mineral vitamínico e aminoácidos de modo a satisfazer as exigências nutricionais de mantença e de produção da linhagem em estudo, além do produto comercial ${ }^{1}$ contendo o extrato de Panax ginseng que foi suplementado de forma on top durante 30 dias (tabela I). A quantidade de ração distribuída para cada ave foi de $110 \mathrm{~g}$ ave/dia durante 30 dias. Após este período todas as aves passaram a receber a mesma dieta, mas na quantidade de $115 \mathrm{~g}$ de ração/ave/dia.

As dietas foram isoprotéicas e isoenergéticas, e seguiram as tabelas de composição de ingredientes de Rostagno et al. (2005).

Os tratamentos foram constituídos da forma como segue: T1: controle (sem adição de $P$. ginseng); T2: controle $+1,9 \mathrm{mg} P$.

${ }^{1}$ Composição do produto comercial a base de $P$. ginseng: Nitrato de tiamina, $2 \mathrm{mg}$; riboflavina, 2 $\mathrm{mg}$; cloridrato de piridoxina, $6 \mathrm{mg}$; inositol, $5 \mathrm{mg}$; cianocobalamina, $5 \mathrm{mcg}$; pantotenato de cálcio, $10 \mathrm{mg}$; nicotinamida, $15 \mathrm{mg}$; ácido ascórbico, 60 $\mathrm{mg}$; acetato de tocoferol, $10 \mathrm{mg}$; P. ginseng, 200 $\mathrm{mg}$; fosfato de cálcio dibásico, $150 \mathrm{mg}$; sulfato ferroso, $15 \mathrm{mg}$; bitartarato de colina, $10 \mathrm{mg}$; retinol, $7500 \mathrm{UI}$; Acido fólico, 0,20 mg; biotina, 0,25 mg; metionina, $5 \mathrm{mg}$; adenosina, 0,75 mg. 
ginseng (ave/dia); T3: controle $+3,8 \mathrm{mg} P$. ginseng (ave/dia); T4: controle $+5,7 \mathrm{mg} P$. ginseng (ave/dia); T5: controle + 7,6 $\mathrm{mg} P$. ginseng (ave/dia). O delineamento experimental adotado foi o inteiramente ao acaso, com cinco tratamentos e sete repetições, totalizando 35 unidades experimentais. Cada unidade experimental foi composta por uma gaiola com duas poedeiras.

\section{Coletadesangue}

As coletas de sangue foram realizadas no período da manhã, através de venopunção da veia braquial de 20 aves, sendo quatro repetições por tratamento. Estas coletas foram realizadas no primeiro, décimo quinto e trigésimo dia do início do experimento que coincidiu com o período da suplementação com $P$. ginseng.

\section{Perfilmetabólico}

Foram coletados $3 \mathrm{~mL}$ de sangue de cada ave e dividido em dois tubos, um sem anticoagulante, para a obtenção do soro sanguíneo, e outro tubo com plasma com antiglicolítico (EDTA $10 \%$ e fluoreto de potássio $12 \%$ - para as análises de glicose) conforme indicado pelo laboratório dos kits reagentes utilizados no experimento.

As amostras de sangue foram centrifugadas a $3500 \mathrm{rpm}$ durante 15 minutos, para obtenção de soro ou plasma, e congeladas a $-18^{\circ} \mathrm{C}$ em eppendorfs previamente identificados, para realização das análises de cálcio, fósforo, glicose, gama glutamil transferase (GGT), albumina, colesterol e ácido úrico.

\section{Perfil hematolóGico}

O sangue coletado foi colocado em tubos eppendorf com anticoagulante (ácido etilenodiaminotetraacético), na proporção de $0,8 \mu \mathrm{l}$ para $0,5 \mathrm{ml}$ de sangue.

A determinação do hematócrito foi realizada através do método do microhematócrito, utilizando-se tubo capilar centrifugado a $1200 \mathrm{rpm}$ por 5 minutos em centrifugas sendo os resultados estimados em porcentagem através de tabelas específicas de microhematócrito. Na contagem de leucócitos, utilizou-se uma amostra de sangue e solução de Natt e Herrick'sb, em uma diluição de 1:200, realizando-se a contagem em câmara de Neubauer, sendo contadas as células nos quadrados peque-nos centrais e o resultado multiplicado por 120 .

Para a contagem diferencial leucocitária, preparou-se um esfregaço sanguíneo em lâminas de vidro, fixado com álcool metílico (Metanol) durante cinco minutos e posteriormente corado com hematoxilina-eosina (Panótipo rápido). As lâminas foram lavadas com água destilada, secadas ao ar livre e os esfregaços foram observados ao microscópio ótico com objetiva de imersão. A contagem leucocitária foi classificatória em

Tabela I. Composição da ração para a fase de postura I. (Composition of experimental diets during the initial laying cycle).

\begin{tabular}{lc}
\hline Ingrediente & $\%$ \\
\hline Milho & 62,83 \\
Farelo de soja & 24,60 \\
Farelo de arroz desengordurado & 1,20 \\
Farinha de ostra & 8,00 \\
Sal iodado & 0,37 \\
Supl. vitamínico mineral e aminoácidos & 3,00 \\
Total & 100 \\
Composição calculada & \\
Energia metabolizável (kcal/kg) & 2702,1 \\
Proteína bruta (\%) & 16,52 \\
Lisina digestível (\%) & 0,749 \\
Met + cis digestível (\%) & 0,488 \\
Cálcio (\%) & 3,822 \\
Fósforo disponível (\%) & 0,380 \\
\hline
\end{tabular}

${ }^{1}$ Níveis de garantia por quilo de produto: núcleo postura; cálcio, $269 \mathrm{~g}$; fósforo, $94 \mathrm{~g}$; vitamina A, 334000 UI; vitamina D3, 67000 UI; vitamina E, 234 $\mathrm{mg}$; vitamina $\mathrm{K} 3,50 \mathrm{mg}$; vitamina $\mathrm{B} 1,54 \mathrm{mg}$; vitamina B2, $147 \mathrm{mg}$; vitamina B6, $100 \mathrm{mg}$; vitamina B12, 400 mcg; niacina, 867 mg; ácido fólico, 24 mg; ácido pantotênico, $334 \mathrm{mg}$; metionina, $34 \mathrm{~g}$; manganês, 2334 mg; zinco, 1667 mg; ferro, 2000 mg; cobre, 334 mg; iodo, 12 mg; selênio, 10,2 mg. 


\section{CATALAN, AVILA, LOPES, MONTAGNER, VARGAS, XAVIEREROLL}

granulares (heterófilos, eosinófilos e basófilos) e não granulares (linfócitos, e monócitos).

\section{AVALIAÇÃODO COMPORTAMENTO DAS AVES}

Para a avaliação do comportamento foi utilizada amostragem instantânea. Nesta técnica o observador registrava qual comportamento que o indivíduo estava realizando em diferentes pontos numa escala de tempo calculando as frequências dos comportamentos.

Os registros foram do tipo instantâneo durando 30 segundos de observação em cada par de gaiolas. Os comportamentos foram considerados mutuamente exclusivos.

Para este estudo as aves foram acompanhadas durante três dias consecutivos. As observações foram realizadas durante duas horas consecutivas no período da manhã e duas horas à tarde, totalizando quatro horas de observação por dia e 287 observações por ave. Essas avaliações foram realizadas no período final de suplementação com $P$. ginseng, isto é, do vigésimo sétimo ao trigésimo dia de experimento. O observador ficou a uma distância de aproximadamente dois metros das aves e atrás da bateria de gaiolas que não estava sendo avaliada para não interferir no comportamento das mesmas.

Os comportamentos analisados foram adaptados da descrição de Hansen (1994), Webster (2000) e Elston et al. (2000), conforme descritos a seguir: comer (qualquer movimento com a cabeça dirigida para dentro do comedouro); Beber (ingestão de água nos bebedouros); Bicagem agressiva (bicadas fortes, arrancando penas, e agressivas dirigidas a outra galinha; Vigiar (dentro da gaiola: a ave em estado de alerta movendo a cabeça de um lado para outro; b) fora da gaiola: a ave em estado de alerta movendo a cabeça de um lado para outro); Caminhar (locomoção com pelo menos um passo em qualquer direção); Sentar (posição de descanso com o peito em contato com o piso da gaiola); Dormir (ave em aparente estado de sonolência com os olhos fechados); Toilette (manipulação suave das próprias penas com o bico).

\section{ANÁLISE ESTATÍSTICA}

Para predizer o efeito das doses de $P$. ginseng sobre os parâmetros metabólicos e hematológicos foi utilizada análise de regressão polinomial. Para comparação dos resultados obtidos com cada nível de inclusão do $P$. ginseng em relação à ração testemunha, foi utilizado o teste Dunnett (5 $\%)$. Para a análise da frequência de comportamentos dentro dos grupos experimentais foi utilizado teste de qui-quadrado ( $\mathrm{p}<0,05$ ). Nos comportamentos em que $50 \%$ dos casos tinham frequências observadas menores que cinco foi utilizado o Teste Exato de Fisher.

\section{RESULTADOS}

Na tabela II observa-se que o perfil hematológico das poedeiras não alterou significativamente com as diferentes doses de $P$. ginseng em nenhum dos períodos analisados.

Os resultados da bioquímica sanguínea são apresentados na tabela III, onde diferenças significativas $(p<0,05)$ entre os tratamentos foram observadas somente para o fósforo em todos os períodos de análise e, para a albumina e o colesterol apenas no terceiro período.

Na avaliação do comportamento, através do teste de qui-quadrado foi possível observar diferença significativa $(\mathrm{p}<0,05)$ nos comportamentos de vigiar com a cabeça para fora da gaiola, toilette ou higiene (manipular e investigar as próprias penas) e beber água. Através do teste exato de Fischer não foi observado diferença entre tratamentos no comportamento agressivo (figura 1).

\section{DISCUSSÃO \\ Perfil hematológico \\ Segundo Macari e Luquetti (2002), a contagem diferencial de leucócitos no}


POEDEIRAS COMERCIAIS SUPLEMENTADAS COM PANAX GINSENG

Tabela II. Perfil hematológico das poedeiras suplementadas com diferentes níveis de Panax ginseng. (Hematological profile of laying hens supplemented with differents levels of Panax ginseng).

\begin{tabular}{|c|c|c|c|c|c|c|c|}
\hline & \multicolumn{5}{|c|}{ Níveis de inclusão de Panax ginseng (mg/dia/ave) } & \multirow[t]{2}{*}{$\mathrm{p}$} & \multirow[t]{2}{*}{ CV (\%) } \\
\hline & 0 & 1,9 & 3,8 & 5,7 & 7,6 & & \\
\hline \multicolumn{8}{|l|}{21 a 22 semanas } \\
\hline Heterófilos (\%) & 23,00 & 23,75 & 24,25 & 22,00 & 22,33 & 0,9913 & 29,71 \\
\hline Linfócitos (\%) & 76,00 & 73,00 & 74,75 & 76,66 & 75,66 & 0,9625 & 9,55 \\
\hline Eosinófilos (\%) & 0,75 & 1,75 & 0,00 & 0,66 & 0,33 & 0,1488 & 126,39 \\
\hline Monócitos (\%) & 0,00 & 0,00 & 0,50 & 0,00 & 0,66 & 0,3275 & 238,98 \\
\hline Basófilos (\%) & 0,25 & 1,50 & 0,50 & 0,66 & 1,00 & 0,6953 & 165,02 \\
\hline Heterófilos/linfócitos (\%) & 0,30 & 0,32 & 0,33 & 0,29 & 0,30 & 0,9882 & 38,90 \\
\hline Leucócitos $(\mu \mathrm{L})$ & 11385 & 31552 & 10415 & 11147 & 9460 & 0,5186 & 147,36 \\
\hline Hematócrito (\%) & 24,50 & 22,60 & 16,33 & 27,33 & 22,25 & 0,4050 & 38,77 \\
\hline \multicolumn{8}{|l|}{23 a 24 semanas } \\
\hline Heterófilos (\%) & 25,66 & 27,00 & 29,60 & 25,60 & 23,00 & 0,3981 & 19,91 \\
\hline Linfócitos (\%) & 74,00 & 71,00 & 69,60 & 73,00 & 75,60 & 0,3725 & 6,96 \\
\hline Eosinófilos (\%) & 0,33 & 0,40 & 1,00 & 0,20 & 0,40 & 0,3457 & 138,11 \\
\hline Monócitos (\%) & 0,16 & 0,80 & 0,20 & 1,00 & 0,60 & 0,4551 & 160,23 \\
\hline Basófilos (\%) & 0,33 & 0,20 & 0,00 & 0,20 & 0,40 & 0,7976 & 234,18 \\
\hline Heterófilos/linfócitos (\%) & 0,35 & 0,38 & 0,43 & 0,35 & 0,30 & 0,3832 & 27,65 \\
\hline Leucócitos $(\mu \mathrm{L})$ & 11740 & 9988 & 8844 & 10648 & 9768 & 0,7649 & 36,33 \\
\hline Hematócrito (\%) & 24,16 & 25,60 & 27,40 & 24,20 & 23,80 & 0,5787 & 15,68 \\
\hline \multicolumn{8}{|l|}{25 a 26 semanas } \\
\hline Heterófilos (\%) & 22 & 22,8 & 25,8 & 22,2 & 21,8 & 0,3695 & 15,15 \\
\hline Linfócitos (\%) & 77,2 & 76,8 & 73,4 & 76,2 & 77,8 & 0,3534 & 4,63 \\
\hline Eosinófilos (\%) & 0,60 & 0,80 & 0,40 & 1,00 & 0,00 & 0,1250 & 107,14 \\
\hline Monócitos (\%) & 0,20 & 0,00 & 0,20 & 0,40 & 0,20 & 0,6974 & 212,13 \\
\hline Basófilos (\%) & 0,00 & 0,00 & 0,20 & 0,20 & 0,20 & 0,7360 & 288,67 \\
\hline Heterófilos/linfócitos (\%) & 0,29 & 0,29 & 0,35 & 0,29 & 0,28 & 0,3710 & 20,10 \\
\hline Leucócitos $(\mu \mathrm{L})$ & 8184 & 8096 & 9284 & 8712 & 8844 & 0,9052 & 25,37 \\
\hline Hematócrito (\%) & 21,60 & 22,20 & 22,80 & 19,40 & 21,80 & 0,7221 & 18,57 \\
\hline
\end{tabular}

sangue de frangos mostra que a proporção normal de heterófilos/linfócitos está em torno de 1/2. Quando frangos são submetidos a condições de estresse essa relação aumenta, pois a quantidade de heterófilos aumenta na circulação. Com isso, podemos afirmar que a relação entre heterófilos/ linfócitos das poedeiras suplementadas com $P$. ginseng esteve dentro da normalidade, indicando por este parâmetro, que a inclusão de $P$. ginseng na dieta não influenciou a condição de estresse das aves nos períodos avaliados. Porém, não se descarta a possibilidade de que estes dados estejam influenciados pelo processo de extração de sangue, que é um procedimento invasivo, levando em consideração que as aves não receberam um período de adaptação para a coleta.

Yan et al. (2011) observaram em frangos de corte, alimentados com raízes de ginseng, resultados muito semelhantes $(75,6 \%)$ para a porcentagem de linfócitos no grupo controle. Porém estes autores observaram um aumento significativo na contagem de linfócitos nos níveis mais elevados de inclusão de ginseng fato este não comprovado no presente estudo. No entanto, é importante ressaltar que os resultados apresentam grande variabilidade conforme observados pelos altos coeficientes de 
CATALAN, AVILA, LOPES, MONTAGNER, VARGAS, XAVIERE ROLL

Tabela III. Perfil bioquímico das poedeiras suplementadas com diferentes níveis de Panax ginseng. (Biochemical profile of laying hens supplemented with different levels of Panax ginseng).

\begin{tabular}{|c|c|c|c|c|c|c|c|}
\hline & \multicolumn{5}{|c|}{ Níveis de inclusão de Panax ginseng (mg/dia/ave) } & \multirow[t]{2}{*}{$\mathrm{p}$} & \multirow[t]{2}{*}{ CV (\%) } \\
\hline & 0 & 1,9 & 3,8 & 5,7 & 7,6 & & \\
\hline \multicolumn{8}{|l|}{21 a 22 semanas } \\
\hline Cálcio (mg/dL) & 13,47 & 13,76 & 14,20 & 14,47 & 12,09 & 0,9038 & 27,18 \\
\hline Fósforo (mg/dL) & 5,93 & $4,35^{\star}$ & 4,96 & 5,44 & 6,44 & 0,0048 & 12,38 \\
\hline Glicose (mg/dL) & 154,14 & 181,03 & 180,17 & 168,89 & 172,31 & 0,6739 & 16,53 \\
\hline GGT(UI/L) & 37,72 & 58,19 & 33,94 & 50,65 & 38,25 & 0,1626 & 33,92 \\
\hline Albumina $(\mathrm{g} / \mathrm{dL})$ & 1,71 & 1,78 & 1,90 & 1,89 & 1,88 & 0,3099 & 7,90 \\
\hline Colesterol (mg/dL) & 128,78 & 105,45 & 121,83 & 106,82 & 166,08 & 0,3463 & 15,53 \\
\hline Ácido úrico (g/dL) & 5,62 & 4,73 & 7,48 & 6,73 & 7,75 & 0,6322 & 48,60 \\
\hline \multicolumn{8}{|l|}{23 a 24 semanas } \\
\hline Cálcio (mg/dL) & 21,77 & 24,96 & 19,16 & 23,36 & 24,40 & 0,0549 & 13,79 \\
\hline Fósforo (mg/dL) & 5,13 & 5,67 & 5,15 & $7,00^{*}$ & $6,82^{*}$ & 0,0061 & 15,22 \\
\hline Glicose (mg/dL) & 164,76 & 191,96 & 172,31 & 190,35 & 177,62 & 0,1695 & 10,86 \\
\hline GGT (UI/L) & 28,45 & 45,69 & 37,50 & 30,17 & 29,74 & 0,7075 & 64,51 \\
\hline Albumina (g/dL) & 1,63 & 1,78 & 1,69 & 1,86 & 1,80 & 0,1422 & 8,35 \\
\hline Colesterol (mg/dL) & 88,91 & 113,97 & 108,54 & 127,46 & 130,34 & 0,2633 & 27,41 \\
\hline Ácido úrico (g/dL) & 6,49 & 5,17 & 5,87 & 6,33 & 6,56 & 0,6040 & 25,32 \\
\hline \multicolumn{8}{|l|}{25 a 26 semanas } \\
\hline Cálcio (mg/dL) & 25,93 & 25,99 & 25,55 & 23,31 & 25,70 & 0,1767 & 10,88 \\
\hline Fósforo (mg/dL) & 7,19 & 7,18 & 7,00 & 6,75 & $9,12^{*}$ & 0,0274 & 23,33 \\
\hline Glicose (mg/dL) & 168,62 & 178,76 & 183,61 & 191,43 & 177,81 & 0,1704 & 11,31 \\
\hline GGT (UI/L) & 85,40 & 49,87 & 49,78 & 60,34 & 47,63 & 0,2198 & 69,63 \\
\hline Albumina (g/dL) & 1,85 & 1,90 & 1,86 & 1,75 & $1,96^{*}$ & 0,0026 & 5,88 \\
\hline Colesterol (mg/dL) & 110,73 & $141,84^{*}$ & $141,82^{*}$ & 101,95 & 129,68 & 0,0049 & 22,12 \\
\hline Acido úrico (g/dL) & 5,61 & 5,55 & 5,15 & 6,08 & 4,83 & 0,7019 & 37,24 \\
\hline
\end{tabular}

GGT = gama glutamiltransferase.

*Difere do grupo controle pelo teste de Dunnett $(p<0,05)$.

variação nos eosinófilos e monócitos. Estes dados podem indicar alguns problemas colaterais de saúde das aves ou fatores externos não controlados no experimento.

Os valores apresentados para os basófilos no primeiro período de análise estão acima dos encontrados por Ribeiro (2007). Segundo Maxwell et al. (1992) citado por Ribeiro (2007) um aumento no número de basófilos é resultado de situações de estresse agudo, onde há risco de vida. Porém, como os resultados do perfil hematológico não apresentaram diferenças significativas entre os tratamentos concluise que o $P$. ginseng não melhorou o bem- estar das aves através deste parâmetro. Além da grande variabilidade dos dados, a redução dos basófilos no terceiro período, reforça a hipótese de que pode ter havido um efeito da manipulação das aves, sobre os parâmetros hematológicos avaliados.

Os leucócitos apresentaram médias relativamente abaixo do esperado. No trabalho de Ribeiro (2007) os valores encontravamse dentro dos limites fisiológicos. O número de leucócitos no sangue dos frangos de corte varia de 12000 a 30000 , porém pode mudar em função do sexo, da idade, das condições de estresse e de doenças (Macari e Luquetti, 2002). 

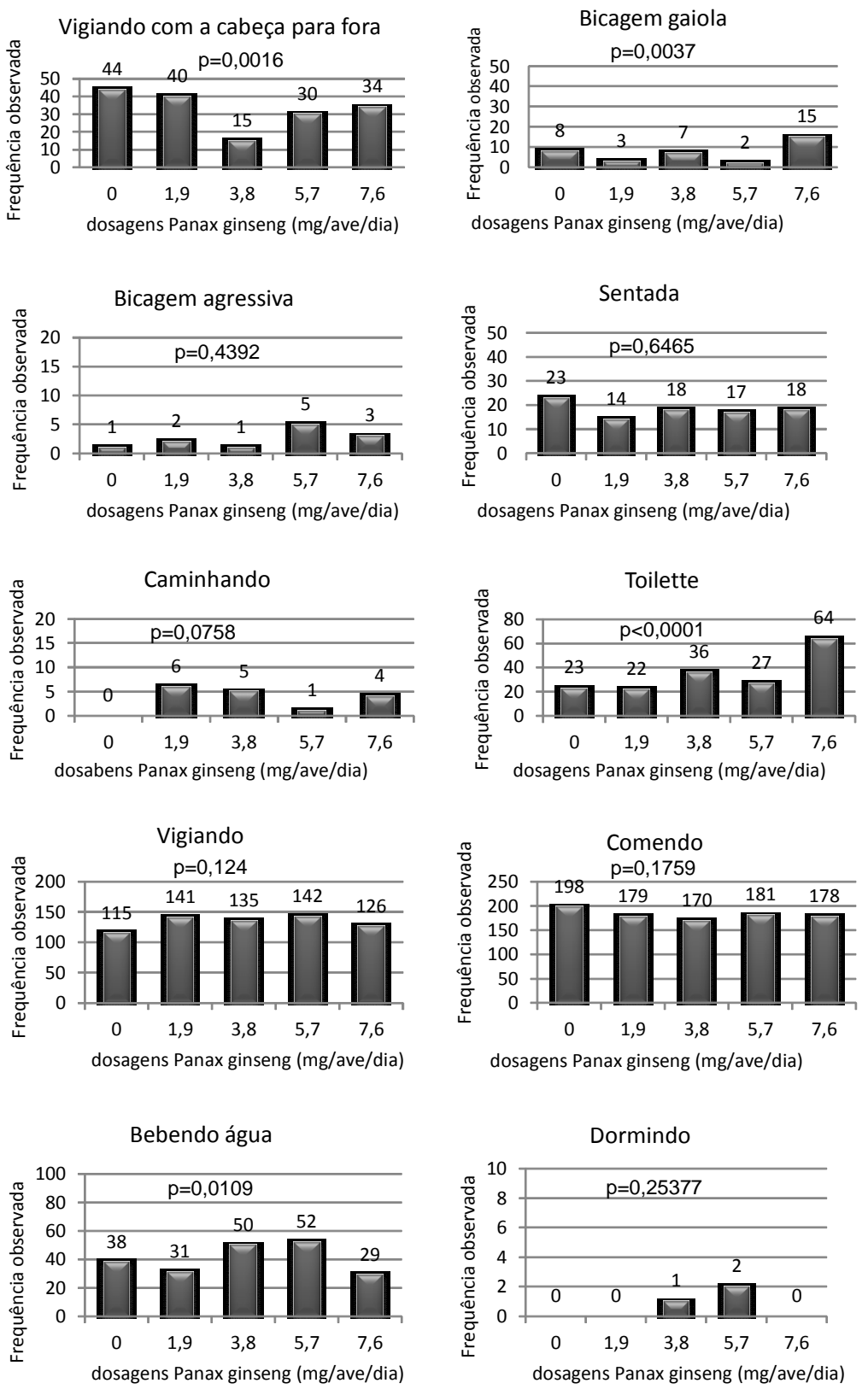

Dados apresentados através da frequência absoluta de acontecimentos (qui-quadrado)

Figura 1. Valores de frequência observada em que as aves expressaram seus comportamentos. (Frequency values observed in hens that expressed their behaviors).

Archivos de zootecnia vol. 62, núm. 237, p. 95. 


\section{CATALAN, AVILA, LOPES, MONTAGNER, VARGAS, XAVIERE ROLL}

Ao comparar com os valores das médias para hematócrito, Ribeiro (2007) suplementou às poedeiras diferentes aditivos $\mathrm{e}$ observou que estes valores são compatíveis com os resultados deste trabalho, com médias em torno de $23 \%$.

Silva et al. (2003) estudaram a influência do sistema de criação de frangos de corte e encontraram valores superiores a estes, com as médias em torno de $30 \%$ de hematócrito. Contudo, os autores salientaram que os menores valores para este parâmetro juntamente com outras variáveis como, maior ganho de peso, menores valores para temperatura retal e taxa respiratória, poderiam indicar conforto e bem-estar às aves.

\section{Perfil BIOQuímico}

As médias para o cálcio encontram-se dentro dos valores apresentados por Gonçalves et al. (2008), e recomendados por Burke (1996) e Schmidt et al. (2007), onde galinhas fora de postura tiveram 9-12 mg/dL de cálcio circulante enquanto que aquelas em postura tiveram $20-40 \mathrm{mg} / \mathrm{dL}$. A maior concentração deste mineral assim como do fósforo, no último período se dá devido à formação da casca do ovo, pois este é derivado da absorção intestinal e da mobilização óssea.

Para o fósforo foi observada um aumento nas concentrações conforme se aproximava do pico de produção das aves, com destaque para as aves que receberam a maior dose de $P$. ginseng que diferiram significativamente do grupo controle no segundo e terceiro períodos de avaliação.

O cálcio e o fósforo são os principais minerais no desenvolvimento das aves, pois estão associados principalmente ao metabolismo à formação óssea e da produção da casca do ovo. A utilização destes minerais pelo organismo depende da idade e do tipo de animal, uma vez que as recomendações nutricionais variam entre as fases de crescimento e de produção (Vargas Jr et al., 2004).

Os níveis de glicose encontrados neste estudo ficaram abaixo do recomendado por Schmidt et al. (2007) onde a concentração sanguínea de glicose de aves sadias variou de 200 a $500 \mathrm{mg} / \mathrm{dL}$. Dentro destes parâmetros apenas no segundo período de coleta os tratamentos com dose de 1,9 e 5,7 $\mathrm{mg} / P$. ginseng/ave apresentaram médias próximas ao padrão, 191,96 mg/dL e 190,35 $\mathrm{mg} / \mathrm{dL}$, respectivamente, e no terceiro período a dose 5,7 $\mathrm{mg}$ de $P$. ginseng com 191,43 $\mathrm{mg} / \mathrm{dL}$ de glicose, que embora crescentes com as doses, não apresentaram diferença estatística entre os tratamentos.

Alguns estudos in vitro e em modelos animais indicaram que as espécies de ginseng asiático $(P$. ginseng) e americano (P. quinquefolious) melhoram o metabolismo de carboidratos. Em relação a isso o autêntico ginseng asiático mostrou efeito hipoglicêmico em animais normais ou hiperglicêmicos (Kimuru e Suzuki, 1991; Martinez e Staba, 1984).

No entanto, os resultados de Sievenpiper et al. (2003) mostraram que $P$. ginseng não teve efeito sobre os valores de glicose no plasma em humanos após a aplicação de um teste oral de tolerância a glicose comparado com um grupo placebo. Respeitando-se a diferença entre espécies estes resultados concordam com o efeito nulo encontrado neste trabalho em poedeiras.

Segundo Radin (2003) a gama glutamiltransferase (GGT) é encontrada no fígado, pâncreas, rim e intestino, contudo as elevações nas concentrações séricas são habitualmente provocadas pelo aumento da produção hepática, pois para González e Silva (2006) somente aquela de origem hepática é normalmente encontrada no plasma, porque a de origem renal é excretada na urina. Os autores também salientam que a função da GGT não está muito bem esclarecida, mas acredita-se que está relacionada com o metabolismo do glutation e pode indicar colestases e proliferação de ductos biliares no fígado de aves.

No trabalho realizado por Gonçalves et al. (2010) os valores encontrados para aves 
no pico de produção era em torno de 17,37 U/L, abaixo do que foi apresentado neste trabalho, sendo que o tratamento controle no último período apresentou a maior média, acima do que foi encontrado por Gonçalves etal. (2010) também em poedeiras comerciais. Porém em nenhum momento houve efeito significativo do $P$. ginseng sobre esta variável.

O incremento das proteínas plasmáticas em poedeiras pode ser explicado pela exigência proteica para formação do ovo, o qual é constituído em média por 12,5\% de proteína (Gonçalves et al., 2008). No segundo período de coleta as médias para albumina foram reduzidas, mas estão dentro do esperado, onde os níveis normais variam de 0,8 a 2,0 g/dL (Schmidt et al., 2007). De um modo geral todos os valores foram semelhantes aos encontrados por Gonçalves et al. (2008) de 2,43 g/dL no pré-pico de produção. No terceiro período houve diferença significativa, onde a maior dosagem de $P$. ginseng (7,6 mg/ave/dia) apresentou valores superiores ao tratamento controle.

Os níveis de colesterol sérico se mantiveram dentro dos valores encontrados por Lumeij et al. (1997) citado por Schmidt et al. (2007) e Gonçalves et al. (2008) que salientam que o colesterol é prontamente transferido do sangue para a formação da gema do ovo. Desta forma, os níveis de colesterol sérico são influenciados pelo momento fisiológico reprodutivo em que a ave se encontra. Neste estudo foi possível observar diferença significativa no terceiro período de análise, sendo que nas dosagens de 1,9 e 3,8 mg/ave/dia foram superiores quando comparados ao controle.

Jang et al. (2007) avaliando os efeitos da suplementação de um subproduto fermentado a base de ginseng, nas quantidades de 2,5 e $5 \%$, em poedeiras comerciais com 55 semanas de idade, sugeriram uma redução do colesterol com a utilização desse subproduto na dieta. Esses resultados discordam com os encontrados na tabela III onde se observa aumento nos níveis de colesterol com a utilização do $P$. ginseng nas dosagens de 1,9 e $3,8 \mathrm{mg} / \mathrm{ave} / \mathrm{dia}$ comparados ao grupo controle somente no terceiro período. Portanto, não houve uma associação entre o aumento das doses de $P$. ginseng com aumento do colesterol total. Este efeito do $P$. ginseng sobre o metabolismo do colesterol é difícil de ser explicado com base na literatura, o que justifica a realização de estudos mais específicos na área levando em conta também a mensuração de colesterol HDL e LDL.

Porém, Yan et al. (2011) observaram redução dos níveis de colesterol em frangos de corte com a utilização de farinha de raízes adventícias de ginseng selvagem comparado ao grupo controle.

As aves são animais uricotélicos, ou seja, excretam o nitrogênio $(\mathrm{N})$ em pequenos volumes de água mediante o ácido úrico como principal produto do metabolismo de $\mathrm{N}$, constituindo $60-80 \%$ do $\mathrm{N}$ excretado na excreta, enquanto que em outras espécies o produto excretado é a uréia, sendo este um bom indicador da função renal das aves (Gonzáleze Silva, 2006; Schmidt et al., 2007). Neste experimento os valores máximos de ácido úrico são semelhantes aos encontrados por Gonçalves et al. (2008) e não variam com a adição de $P$. ginseng na dieta.

\section{COMPORTAMENTO ANIMAL}

Nos resultados de comportamento (figura 1) observa-se que as aves passaram a maior parte do tempo comendo, bebendo água, vigiando e vigiando com a cabeça para fora da gaiola, demonstrando um estado de alerta. Como era de se esperar em gaiolas convencionais de postura os demais comportamentos do seu repertório foram bem menos frequentes. Barbosa Filho et al. (2007) avaliando diferentes sistemas de criação de aves sob estresse ou conforto térmico puderam observar que em condições de estresse térmico, os comportamentos detectados com maior frequência foram os de sentar-se, ficar parada e o de beber água. Portanto, os dados apresentados neste 


\section{CATALAN, AVILA, LOPES, MONTAGNER, VARGAS, XAVIERE ROLL}

estudo indicam que as aves não estavam passando por estresse térmico já que os comportamentos assinalados foram poucos frequentes.

O comportamento de vigiar com a cabeça para fora da gaiola foi significativamente afetado pelos tratamentos. O maior desvio ocorreu na dosagem de $3,8 \mathrm{mg} / P$. ginseng/ dia onde a frequência observada de aves realizando este comportamento esteve bem abaixo da esperada. Este resultado é difícil de explicar, mas pode estar influenciado pela presença de alguns indivíduos com comportamentos individuais repetitivos dentro do tratamento, tendo em vista que as aves foram reamostradas diversas vezes.

De maneira geral no grupo controle e na dose mais baixa de $P$. ginseng a frequência observada de aves em estado de alerta e vigilantes esteve acima da esperada enquanto que nas doses mais altas este comportamento esteve dentro do esperado.

O comportamento de higiene ou toilette foi afetado significativamente pelos tratamentos. Neste caso a frequência observada deste comportamento foi o dobro da esperada nas aves que receberam a maior dose de $P$. ginseng comparado com o grupo controle, mas não com as doses intermediárias. O resultado desta análise indica que somente em doses maiores o $P$. ginseng pode influenciar positivamente o comportamento das poedeiras, pois a higiene das penas está relacionada normalmente com estado de conforto e bem-estar animal. Da mesma forma a frequência do comportamento de bicar partes da gaiola foi significativamente maior nas galinhas suplementadas com a maior dose de $P$. ginseng $(\mathrm{p}=0,0037)$ e não nas doses intermediárias. Isto significa que alguns comportamentos necessitariam de uma dose superior a $5,7 \mathrm{mg} / \mathrm{dia} /$ ave de $P$. ginseng para serem alterados. Este resultado pode indicar que estas galinhas estavam mais dispostas a explorar o ambiente em que vivem mesmo considerando a falta de estímulos inerente deste sistema de criação.

No comportamento de beber verificou- se que a frequência observada de aves bebendo água foi menor nas aves recebendo a maior dose diária de $P$. ginseng, porém foram maiores nas doses 3,8 e $5,7 \mathrm{mg} / \mathrm{dia} /$ ave. Este pode ser um indicativo de que estas aves sofriam menos de estresse térmico, tendo em vista que todas as aves estavam submetidas as mesmas condições ambientais e recebiam a mesma dieta basal. Porém, não se descarta a hipótese de que outros fatores possam ter interferido nos resultados, como por exemplo, o próprio método de amostragem do comportamento, que por ser instantâneo, muitas vezes não representa bem os comportamentos que são executados em uma sequência de eventos, como é o caso de beber água e por esta razão são vistos com menos frequência em outros momentos do dia. Este último comportamento de estar junto ao bebedouro foi observado por Sevegnani et al. (2005) em frangos de corte submetidos a estresse térmico, sendo que a ocorrência deste se fazia pela necessidade de refrigerar o organismo.

Wang et al. (2010) estudaram a atividade antifadiga de polissacarídeos solúveis em água extraídos de $P$. ginseng, através de um teste de natação forçada em camundongos. Estes polissacarídeos contem uma porção denominada neutra composta por uma mistura de glucanos e arabinogalactanos e uma porção acídica que contém arabinogalactanos, pectinas rhamnogalacturonas tipo I e pectinas homogalacturanas (Zhang et al., 2009). Wang et al. (2010) observaram que a administração oral destes polissacarídeos reduziram o tempo de imobilidade após o teste de natação forçada indicando um efeito anti-fadiga que poderia ser explicado provavelmente através de proteção da membrana corpuscular impedindo a oxidação lipídica pela modificação da atividade de várias enzimas. Chen et al. (2008) classificam resumidamente as espécies de $P$. ginseng como estimulante e revigorante e o $P$. quinquefolium como calmante e nutritivo.

Em suma, as variáveis analisadas não apresentaram tendências claras, sendo ob- 
servadas apenas pequenas variações no perfil metabólico e na frequência de alguns comportamentos. A partir deste trabalho é possível concluir que a suplementação com

\section{BIBLIOGRAFIA}

Barbosa Filho, J.A.D.; Silva, I.J.O.; Silva, M.A.N. e Silva, C.J.M. 2007. Avaliação dos comportamentos de aves poedeiras utilizando sequência de imagens. Eng Agric, 27: 93-99.

Burke, W. 1996. Reprodução das aves. In: Dukes, M.J.S. (Ed.). Fisiologia dos animais domésticos. Cap. 41. Editora Guanabara. Rio de Janeiro/RJ. pp. $660-680$.

Chen, C.F.; Chiou, W.F. and Zhang, J.T. 2008. Comparison of the pharmacological effects of Panax ginseng and Panax quinquefolium. Acta Pharm Sinic, 29: 1103-1108.

Choi, K.T. 2008. Botanical characteristics, pharmacological effects and medicinal components of Korean Panax ginseng C.A. Meyer. Acta Pharm Sinic, 29: 1109-1118.

Elston, J.J.; Beck, M.M.; Kachman, S.D. and Scheideler, S.E. 2000. Laying hen behaviour. Effect of cage type and startle stimuli. Poultry Sci, 79: 471-476.

Gonçalves, F.M.; França, R.T.; Dallmann, H.M.; Gentilini, F.P.; Del Pino, F.A.B.; Stercken, R.A.C. e Zanusso, J.T. 2008. Perfil metabólico de poedeiras semipesadas em fase de pré-pico de postura. $45^{\underline{a}}$ Reunião Anual da SBZ. 22-25 de Julho. Anais... Lavras.

Gonçalves, F.M.; Ribeiro, E.M.; Montagner, P.; Lopes, M.S.; Anciuti, M.A.; Gentilini, F.P.; Del Pino, F.A.B. e Correa, M.N. 2010. Níveis séricos de enzimas hepáticas em poedeiras comerciais no pré-pico e pico de produção de ovos. Acta Sci Vet, 38: 311-314.

Gonzáles, F.H.D. e Silva, S.C. 2006. Introdução à Bioquímica Clínica Veterinária. Editora UFRGS. Porto Alegre/RS. 360 pp.

Hansen, I. 1994. Behavioural expression of laying hens in aviary and cages: frequencies, time budgets and facility utilization. Brit Poultry Sci, 35: 491-508.

Jang, H.D.; Kim, H.J.; Cho, J.H.; Chen, Y.J.; Yoo, J.S.; Min, B.J.; Park, J.C. and Kim, I.H. 2007. Effects of dietary supplementation of fermented wild-ginseng culture by-products on egg
$P$. ginseng até a dose de 7,6 mg/ave/dia não é recomendada, pois não altera o desempenho, o perfil hematológico e o status imunológico de poedeiras Hisex Brown.

productivity, egg quality, blood characteristics and ginsenoside concentration of yolk in laying hens. Korean J Poultry Sci, 34: 271-278.

Kiefer, D. and Pantuso, T. 2003. Panax ginseng. Am Fam Physician, 68: 1539-1542.

Kimuru, M. and Suzuki, J. 1991. The pattern of action of blended Chinese traditional medicines to glucose tolerance curves in genetically diabetic mice. J Pharmacobio-Dynam, 4: 907-915.

Lee, M.H.; Lee, S.H.; Kim, Y.J.; Ko, Y.H.; Jang, I.S.; Moon, Y.S.; Choi, Y.-H. and Sohn, S.H. 2008. Effect of dietary anti-oxidant supplementation on telomere length and egg quality in laying hens. Korean J Poultry Sci, 35: 267-274.

Macari, M. y Luquetti, B.C. 2002. Fisiologia cardiovascular. In: Fisiologia aviária: aplicada a frangos de corte. Cap. 2. Editora FUNEP. Jaboticabal/SP. pp. 17-36.

Martinez, B. and Staba, E.J. 1984. The physiological effects of Aralia, Panax and Leutherococcus on exercised rats. Jpn J Pharmacol, 35: 79-85.

Radin, M.J. 2003. Interpretação de perfis bioquímicos. In: Consulta rápida em clínica veterinária. Cap. 13. Editora Guanabara. Rio de Janeiro/RJ. pp. 120-128.

Park, J.H.; Shin, O.S. and Ryu, K.S. 2005. Effect of feeding wild ginseng culture by-products on performance and egg quality of laying hens. Korean J Poultry Sci, 32: 269-273.

Ribeiro, C.L.G. 2007. Efeito da utilização da mananoligossacarídeos (MOS) e de ácidos orgânicos associados à MOS, com e sem antibiótico, na dieta de poedeiras produtoras de ovos avermelhados. Tese (Doutorado) Zootecnia. Universidade Federal de Pelotas. Pelotas. $111 \mathrm{pp}$.

Rostagno, S.H.; Albino, L.F.T.; Donzele, J.L.; Gomes, P.C.; Oliveira, R.F.; Lopes, D.C.; Ferreira, A.S. e Barreto, S.L.T. 2005. Tabelas brasileiras para aves e suínos: composição de alimentos e exigências nutricionais. Editora UFV. Viçosa/ MG. 186 pp.

Schmidt, E.M.S.; Locatelli-Dittrich, R.; Santin, E. e 


\section{CATALAN, AVILA, LOPES, MONTAGNER, VARGAS, XAVIERE ROLL}

Paulillo, A.C. 2007. Patologia clínica em aves de produção -uma ferramenta para monitorar a sanidade avícola- Revisão. Arch Vet Sci, 12: 9 20.

Sevegnani, K.B.; Caro, I.W.; Pandorfi, H.; Silva, I.J.O. e Moura, D.J.2005. Zootecnia de precisão: análise de imagens no estudo do comportamento de frangos de corte em estresse térmico. Rev Bras Eng Agric Amb, 9: 115-119.

Sievenpiper, J.L.; Arnason, J.T.; Leiter, L.A. and Vuksan, V. 2003. Null and opposing effects of Asian ginseng (Panax ginseng C.A. Meyer) on acute glycemia: results of two acute dose escalation studies. J Am Coll Nutr, 22: 524-532.

Silva, M.A.N.; Hellmeister Filho, P.; Rosário, M.F.; Coelho, A.A.D.; Savino, V.J.M.; Garcia, A.A.F.; Silva, I.J.O. e Menten, J.F.M. 2003. Influência do sistema de criação sobre o desempenho, a condição fisiológica e comportamento de linhagens de frangos de corte. Rev Bras Zootecn, 32: 208-213.

Vargas Junior, J.G.; Albino, L.F.T.; Rostagno, H.S.; Gomes, P.C.; Cupertino, E.S.; Carvalho, D.C.O.; Silva, M.A. e Pinto, R. 2004. Níveis nutricionais de cálcio e de fósforo disponível para aves de reposição leves e semipesadas de 7 a 12 semanas de idade. Rev Bras Zootecn, 33: 936-946.

Yan, L.; Meng, Q.W.; Lee, J.H.; Wang, J.P. and Kim, I.H. 2011. Effects of dietary wild-ginseng adventitious root meal on growth performance, blood profiles, relative organ weight and meat quality in broiler chickens. Asian Austral J Anim, 24: 258.

Wang, J.; Li, S.; Fan, Y.; Chen, Y.; Liu, D.; Cheng, H.; Gao, X. and Zhou, Y. 2010. Anti-fatigue activity of the water-soluble polysaccharides isolated from Panax ginseng C. A. Meyer. J Ethnopharmacol, 130: 421-423.

Webster, A.B. 2000. Behaviour of White Leghorn hens after withdrawal of feed. Poultry Sci, 79: 192-200.

Zhang, X.; Yu, L.; Bi, H.T.; Li, X.H.; Ni, W.H.; Han H.; Li, N.; Wang, B.Q.; Zhou, Y.F. and Tai, G.H. 2009. Total fractionation and characterization of the water-soluble polysaccharides isolated from Panax ginseng C.A. Meyer. Carbohyd Polym, 7: 544-552. 